\title{
En France...
}

\section{La médaille Anton Bruun pour Véronique Garçon}

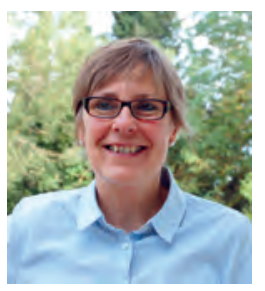

Lors de la $29^{\mathrm{e}}$ assemblée de la Commission océanographique internationale (IOC de l'Unesco), qui s'est déroulée en juin 2017 à

Paris, Véronique Garçon s'est vue décerner la médaille Anton Bruun, du nom de l'océanographe et ichtyologiste danois Anton Frederik Bruun (1901-1961). Ce prix récompense chaque année depuis 2005 les travaux d'un océanographe de renom. Véronique est directrice de recherche au CNRS, affiliée au Laboratoire d'études en géophysique et océanographie spatiales (Legos) à Toulouse. Ses recherches contribuent à mieux comprendre les flux des composés biogéochimiques dans l'océan aux échelles globale et régionale à partir d'observations satellitaires et in situ, et d'outils de modélisation. Ces dernières années, Véronique a focalisé une partie de son travail sur la désoxygénation des océans qui a un impact sur les habitats et écosystèmes marins et sur les cycles biogéochimiques. Elle a montré par exemple l'importance de la circulation océanique, y compris de méso-échelle, dans le maintien des zones de minimum d'oxygène dans les régions de courant de bord est. Enfin, Véronique est aujourd'hui Chair du programme Solas ${ }^{1}$ et membre du Global Ocean Oxygen Network ${ }^{2}\left(\mathrm{GO}_{2} \mathrm{NE}\right)$ de l'IOC de l'Unesco.

1. Surface Ocean Lower Atmosphere Study, www.solas-int.org

2.www.unesco.org/new/en/natural-sciences/ ioc-oceans/sections-and-programmes/oceansciences/global-ocean-oxygen-network/

\section{En France...}

\section{Deux colloques concernant les " services climatiques »}

Deux colloques en lien avec les « services climatiques ${ }^{1}$ » se sont tenus au tout début juin de cette année 2017 à Paris : la Journée utilisateurs des services climatiques et le Colloque de restitution du projet Extremoscope.

Le premier, qui a réuni environ 120 participants, était co-organisé le $1^{\mathrm{er}}$ juin sur le site de l'université Pierre-etMarie-Curie à Jussieu par AllEnvi (Alliance nationale de recherche pour l'environnement) et le ministère de la Transition écologique et solidaire. Le principal objectif de cette journée était de recueillir une expression de besoins de la part des utilisateurs des services climatiques et leurs avis sur les axes de développement déjà identifiées pour les deux ou trois prochaines années. Plus particulièrement, il s'agissait d'infléchir le développement de trois services déjà existants :

- Drias, ouvert en 2012, permet d'accéder aux projections climatiques régionalisées réalisées dans les laboratoires français de modélisation du climat (IPSL, Cerfacs, CNRM) : www.drias-climat.fr/ (figure 1);

- IPSL-CSE, issu de l'Institut Pierre-Simon Laplace (IPSL), a entre autres pour mission de développer la

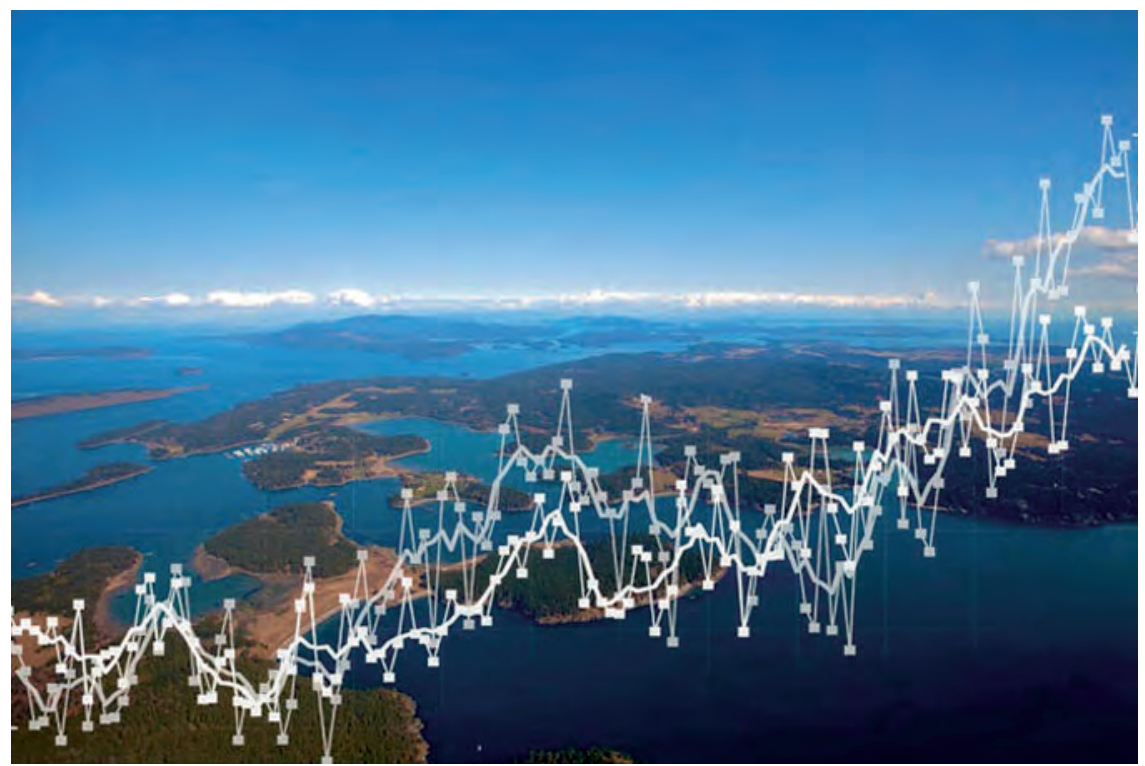

Figure 1. Une image de « l'espace d'accompagnement » du site Drias.

valorisation, la diffusion et le transfert des simulations climatiques pour les besoins de l'adaptation et de l'atténuation du changement climatique : http://cse.ipsl.fr/;

- ClimatHD, application ouverte en 2015 par Météo-France, propose une approche intégrée et simple du diagnostic du changement climatique, à la fois sur le climat passé et futur, mais également à l'échelle nationale et régionale : www.meteofrance.fr/climatpasse-et-futur/climathd.

Après une présentation de Jean-Noël Thépaut du Centre européen pour les prévisions météorologiques à moyen terme (CEPMMT) permettant de situer 
les liens de ces services avec l'initiative européenne Copernicus Climate Change Service (C3S), la matinée était consacrée à une présentation de chaque service suivie par une discussion avec la salle. Chaque présentation était à « double voix » puisqu'elle associait un porteur ou une porteuse du service à un utilisateur ou une utilisatrice exprimant l'intérêt mais aussi les limites de l'information aujourd'hui disponible. Les échanges avec la salle ont pu se développer l'après-midi au travers d'ateliers orientés d'une part sur les contenus mis à disposition, d'autre part sur les fonctionnalités et l'accompagnement des utilisateurs.

Comme souligné par Éric Brun, secrétaire général de l'Observatoire national sur les effets du réchauffement climatique (Onerc), dans ses mots de conclusion, les échanges avec la très grande diversité d'utilisateurs publics et privés représentés ont permis de faire remonter de nombreuses questions et propositions qui permettront sûrement d'enrichir les services rendus.

Le lendemain 2 juin, un autre colloque, qui a réuni une cinquantaine de participants, était organisé par le ministère de la Transition écologique et solidaire sur le site de la Direction du CNRS à Paris. Ce colloque a permis quant à lui de définir les premiers contours de ce que pourrait être un service climatique d' « attribution » des événements extrêmes, c'est-à-dire permettant de préciser le lien entre ces événements et le changement climatique anthropique. Concentré sur une demi-journée, il avait en effet pour but de présenter et discuter les principaux résultats du projet de recherche Extremoscope ${ }^{2}$ soutenu de 2013 à 2016 par le ministère.

Les présentations effectuées par les porteurs du projet ont permis d'aborder la question de la détermination du rôle du changement climatique anthropique sur l'occurrence et l'intensité des extrêmes climatiques en France. Tour à tour, les méthodologies mises en place ont été présentées, puis les indicateurs développés pour caractériser et suivre l'évolution des événements, et enfin l'application de ces méthodes et indicateurs à six événements majeurs (fortes chaleurs ou forts cumuls de pluie) qui se sont produits et ont été étudiés au cours du projet. Des fiches synthétiques relatives à chaque événement avaient été distribuées aux participants afin qu'ils réagissent à la présentation d'informations sur les extrêmes climatiques dans le cadre d'un futur service de nature « opérationnelle ». Les discussions ont fait ressortir un réel intérêt pour ce type d'information et des propositions à la fois sur les contenus et les modes de diffusion.
Deux sites internet permettent d'ores et déjà d'avoir accès à l'ensemble des présentations de ces deux colloques en attendant que les comptes rendus y soient aussi mis en ligne: www.meteo.fr/ cic/jusc2017/ pour la journée des utilisateurs et www.meteo.fr/cic/ extremoscope2017/ pour la restitution du projet Extremoscope (avec aussi accès aux fiches concernant les extrêmes climatiques récents).

Serge Planton

Centre national de recherches météorologiques, Météo-France, Toulouse

Robert Vautard

Laboratoire des sciences du climat et de l'environnement,

CEA / CNRS / UVSQ, Gif-sur-Yvette

1. « Ensemble des informations et prestations qui permettent d'évaluer et de qualifier le climat passé, présent ou futur, d'apprécier la vulnérabilité des activités économiques, de l'environnement et de la société au changement climatique, et de fournir des éléments pour entreprendre des mesures d'atténuation et d'adaptation. »

Source : Stratégie scientifique de développement des services climatiques, AllEnvi, 2014.

2. Interprétation et attribution des événements météorologiques et climatiques extrêmes dans un cadre climatique en France.

\section{En France...}

\section{Un drone au-dessus des océans : le projet Miriad}

Le Centre national de recherches météorologiques (CNRM) de MétéoFrance est porteur d'un projet dédié à l'étude de la couche limite marine de l'atmosphère à l'aide de drones instrumentés : Miriad (système de mesures scientifiques de flux de surface en milieu maritime embarqué sur drone). Il est mené en partenariat avec le Laboratoire d'aérologie (LA) de l'Observatoire Midi-Pyrénées et la société toulousaine Boréal ${ }^{1}$, et est soutenu par la région Occitanie et l’Union européenne.

1. Boréal Systèmes est une société privée de la région toulousaine, membre du pôle Aerospace Valley, spécialisée dans le développement et la construction de drones à grande capacité.
Les aérosols jouent un rôle important sur le climat. Ils influencent les propriétés radiatives de l'atmosphère en diffusant et absorbant les rayons du soleil (effet direct) et en modifiant les propriétés microphysiques et optiques des nuages (effet indirect) parce qu'ils servent de noyaux de condensation indispensables à la formation des hydrométéores. Les océans couvrent plus de $70 \%$ de la surface de la Terre, mais les émissions d'aérosols marins sont encore mal quantifiées. Pourtant, une meilleure connaissance de leur contribution est indispensable pour comprendre les variations naturelles de la composition de l'atmosphère et leurs rétroactions, afin d'évaluer le rôle des activités humaines sur le climat. Par conséquent, les océans sont des régions clés pour les mesures de flux de surface d'énergie et d'aérosols qui permettront d'améliorer les modèles météorologiques et climatiques. En particulier, nombre d'études montrent que, pour évaluer l'effet climatique des aérosols marins, leurs sources et leurs puits doivent être correctement évalués. La concentration en aérosols dans les couches limites marines étant faible, les nuages sont plus sensibles à leurs effets indirects que dans les régions continentales. L'importance relative des sources locales de production d'aérosols à la surface de la mer (provenant du déferlement des vagues, couverture moutonneuse) par rapport à la concentration de fond demeure une 